Afrika Statistika

Afrika Statistika

Vol. 9, 2014, pages 615-625.

DOI: http://dx.doi.org/1016929.as.2014.615.57

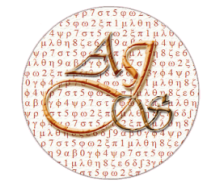

ISSN 2316-090X

\title{
On drift estimation for non-ergodic fractional Ornstein-Uhlenbeck process with discrete observations
}

\author{
Khalifa Es-Sebaiy ${ }^{\dagger, *}$ and Djibril Ndiaye $\mathrm{e}^{\ddagger, 1}$ \\ ${ }^{\dagger}$ National School of Applied Sciences - Marrakesh, Cadi Ayyad University, Marrakesh, Morocco \\ ${ }^{\ddagger}$ Laboratoire de Mathématiques Appliquées, Université Cheikh Anta Diop De Dakar BP 5005 Dakar- \\ Fann Sénégal
}

Received 26 Mai 2014; Accepted 16 October 2014

Copyright (c) 2014, Afrika Statistika. All rights reserved

\begin{abstract}
We consider parameter estimation problems for the non-ergodic fractional Ornstein-Uhlenbeck process defined as $d X_{t}=\theta X_{t} d t+d B_{t}^{H}, t \geq 0$, with an unknown parameter $\theta>0$, where $B^{H}$ is a fractional Brownian motion of Hurst index $H \in\left(\frac{1}{2}, 1\right)$. We assume that the process $\left\{X_{t}, t \geq 0\right\}$ is observed at discrete time instants $t_{1}=\Delta_{n}, \ldots, t_{n}=n \Delta_{n}$. We construct two estimators $\hat{\theta}_{n}$ and $\check{\theta}_{n}$ of $\theta$ which are strongly consistent, namely, $\hat{\theta}_{n}$ and $\check{\theta}_{n}$ converge to $\theta$ almost surely as $n \rightarrow \infty$. We also prove that $\sqrt{n \Delta_{n}}\left(\hat{\theta}_{n}-\theta\right)$ and $\sqrt{n \Delta_{n}}\left(\check{\theta}_{n}-\theta\right)$ are tight.

Résumé. Dans ce travail, nous étudions des problèmes d'estimation paramétriques relatifs au processus d'Ornstein-Uhlenbeck fractionaire non-ergodique défini par $d X_{t}=\theta X_{t} d t+$ $d B_{t}^{H}, t \geq 0$, où $\theta>0$ est un paramètre et $B^{H}$ est un mouvement Brownien fractionaire d'indice de Hurst $H \in] 1 / 2,1\left[\right.$. Le processus $\left\{X_{t}, t \geq 0\right\}$ a été observé (de façon régulière) aux instants $t_{1}=\Delta_{n}, \ldots, t_{n}=n \Delta_{n}$, c'est-à-dire pour tout $i \in\{0, \cdots, n\}, t_{i}=i \Delta_{n}$. Nous avons construit deux estimateurs $\hat{\theta}_{n}$ et $\check{\theta}_{n}$ de $\theta$ fortement consistants, c'est-à-dire, $\hat{\theta}_{n}$ et $\check{\theta}_{n}$ convergent presque surement vers $\theta$ quand $n \rightarrow \infty$. Nous avons aussi prouvé que $\sqrt{n \Delta_{n}}\left(\hat{\theta}_{n}-\theta\right)$ et $\sqrt{n \Delta_{n}}\left(\check{\theta}_{n}-\theta\right)$ sont tendus.
\end{abstract}

Key words: Drift estimation; Discrete observations; Ornstein-Uhlenbeck process; Nonergodicity.

AMS 2010 Mathematics Subject Classification : 60G22; 62M05; 62F12.

\footnotetext{
*Corresponding author Khalifa Es-Sebaiy: k.Essebaiy@uca.ma

Djibril Ndiaye : djibykhady@yahoo.fr

${ }^{1}$ Supported by "La commission de l'UEMOA dans le projet PACER II signé avec le département de mathématiques et informatique de l'UCAD"
} 
K. Es-Sebaiy and D. Ndiaye, Afrika Statistika, Vol. 9, 2014, pages 615-625. On drift estimation for non-ergodic fractional Ornstein-Uhlenbeck process with discrete observations.

\section{Introduction}

Consider the Ornstein-Uhlenbeck process $X=\left\{X_{t}, t \geq 0\right\}$ defined as

$$
X_{0}=0, \quad \text { and } \quad d X_{t}=\theta X_{t} d t+d B_{t}^{H}, t \geq 0
$$

where $B^{H}=\left\{B_{t}^{H}, t \geq 0\right\}$ is a fractional Brownian motion of Hurst index $H>\frac{1}{2}$ and $\theta \in(-\infty, \infty)$ is an unknown parameter. An interesting problem is to estimate the parameter $\theta$ when one observes the whole trajectory of $X$.constant

In the continuous case, recently, by using the least squares estimator (LSE) $\tilde{\theta}_{t}$ of $\theta$ given by

$$
\tilde{\theta}_{t}=\frac{\int_{0}^{t} X_{s} d X_{s}}{\int_{0}^{t} X_{s}^{2} d s}, \quad t \geq 0,
$$

$\mathrm{Hu}$ and Nualart (2010) and Belfadli et al. (2011) have studied the consistency and the asymptotic distributions of $\tilde{\theta}_{t}$ based on the observation $\left\{X_{t}, t \in[0, T]\right\}$ as $T \rightarrow \infty$.

The LSE $\tilde{\theta}_{t}$ is obtained by the least squares technique, that is, $\tilde{\theta}_{t}$ (formally) minimizes

$$
\theta \longmapsto \int_{0}^{t}\left|\dot{X}_{s}-\theta X_{s}\right|^{2} d s
$$

To obtain the consistency of the LSE $\tilde{\theta}_{t}$, in the recurrent case corresponding to $\theta<0, \mathrm{Hu}$ and Nualart (2010) are forced to consider $\int_{0}^{t} X_{s} d X_{s}$ as a Skorohod integral rather than an integral in a path-wise sense. Assuming $\int_{0}^{t} X_{s} d X_{s}$ is a Skorohod integral and $\theta<0$, they proved the strong consistence of $\tilde{\theta}_{t}$ if $H \geq \frac{1}{2}$, and that the LSE $\tilde{\theta}_{t}$ is asymptotically normal if $H \in\left[\frac{1}{2}, \frac{3}{4}\right)$. In the non-recurrent case corresponding to $\theta>0$, Belfadli et al. (2011) established, when $H>\frac{1}{2}$, that the LSE $\tilde{\theta}_{t}$ of $\theta$ is strongly consistent and asymptotically Cauchy, where in their case, the integral $\int_{0}^{t} X_{s} d X_{s}$ is interpreted as an integral in a pathwise sense. The almost sure central limit theorem (ASCLT) for the estimator $\tilde{\theta}_{t}$, in the case when $\theta<0$, is also studied by Cénac and Es-Sebaiy (2012). They proved that, when $H \in(1 / 2,3 / 4)$, the sequence $\left\{\sqrt{n}\left(\theta-\tilde{\theta}_{n}\right)\right\}_{n \geq 1}$ satisfies the ASCLT.

From a practical point of view, in parametric inference, it is more realistic and interesting to consider asymptotic estimation for $X$ based on discrete observations.

Assume that the process $X$ is observed equidistantly in time with the step size $\Delta_{n}: t_{i}=$ $i \Delta_{n}, i=0, \ldots, n$, and $T_{n}=n \Delta_{n}$ denotes the length of the 'observation window'. The purpose of this paper, when $\theta>0$ corresponding to the non-recurrent case, is to construct two estimators for $\theta$ converging at rate $\sqrt{n \Delta_{n}}$ based on the sampling data $X_{t_{i}}, i=0, \ldots, n$.

Suppose that the integral $\int_{0}^{t} X_{s} d X_{s}$ is interpreted in the Young sense (path-wise sense). Then we can write

$$
\tilde{\theta}_{T_{n}}=\frac{\int_{0}^{T_{n}} X_{s} d X_{s}}{\int_{0}^{T_{n}} X_{s}^{2} d s}=\frac{X_{T_{n}}^{2}}{2 \int_{0}^{T_{n}} X_{s}^{2} d s} .
$$


Now, let us construct two discrete versions of $\tilde{\theta}_{T_{n}}$. If, in (2), $d X_{s}$ is replaced by $\left(X_{t_{i}}-X_{t_{i-1}}\right)$, and $\int_{0}^{T_{n}} X_{s}^{2} d s$ by $\Delta_{n} \sum_{i=1}^{n} X_{t_{i-1}}^{2}$, we obtain the following estimators of $\theta$,

$$
\hat{\theta}_{n}=\frac{\sum_{i=1}^{n} X_{t_{i-1}}\left(X_{t_{i}}-X_{t_{i-1}}\right)}{\Delta_{n} \sum_{i=1}^{n} X_{t_{i-1}}^{2}}
$$

and

$$
\check{\theta}_{n}=\frac{X_{t_{n}}^{2}}{2 \Delta_{n} \sum_{i=1}^{n} X_{t_{i-1}}^{2}} .
$$

For non-ergodic diffusion processes driven by Brownian motion based on discrete observations, parametric estimation problems have been studied for instance by Jacod (2006), Dietz and Kutoyants (2003) and Shimizu (2009).

The rest of our paper is organized as follows. In Section 2 we introduce the needed material for our study. In section 3 we prove the strong consistency of $\hat{\theta}_{n}$ and $\check{\theta}_{n}$. Finally, section 4 is devoted to establish that the sequences $\sqrt{n \Delta_{n}}\left(\hat{\theta}_{n}-\theta\right)$ and $\sqrt{n \Delta_{n}}\left(\check{\theta}_{n}-\theta\right)$ are tight.

\section{Basic notions for fractional Brownian motion}

In this section, we briefly recall some basic facts concerning stochastic calculus with respect to a fractional Brownian motion; we refer to Nualart (2006) for further details. Let $B^{H}=$ $\left\{B_{t}^{H}\right\}_{t \in[0, T]}$ be a fractional Brownian motion with Hurst parameter $H \in(0,1)$, defined on some probability space $(\Omega, \mathcal{F}, P)$. (Here, and everywhere else, we do assume that $\mathcal{F}$ is the sigma-field generated by $B^{H}$.) This means that $B^{H}$ is a centered Gaussian process with the covariance function $E\left[B_{s}^{H} B_{t}^{H}\right]=R_{H}(s, t)$, where

$$
R_{H}(s, t)=\frac{1}{2}\left(t^{2 H}+s^{2 H}-|t-s|^{2 H}\right) .
$$

If $H=\frac{1}{2}$, then $B^{\frac{1}{2}}$ is a Brownian motion.

We denote by $\mathcal{E}$ the set of step $\mathbb{R}$-valued functions on $[0, T]$. Let $\mathcal{H}$ be the Hilbert space defined as the closure of $\mathcal{E}$ with respect to the scalar product

$$
\left\langle\mathbf{1}_{[0, t]}, \mathbf{1}_{[0, s]}\right\rangle_{\mathcal{H}}=R_{H}(t, s) .
$$

We denote by $|\cdot|_{\mathcal{H}}$ the associate norm. The mapping $\mathbf{1}_{[0, t]} \mapsto B_{t}^{H}$ can be extended to an isometry between $\mathcal{H}$ and the Gaussian space associated with $B^{H}$. We denote this isometry by

$$
\varphi \mapsto B^{H}(\varphi)=\int_{0}^{T} \varphi(s) d B_{s}^{H}
$$


K. Es-Sebaiy and D. Ndiaye, Afrika Statistika, Vol. 9, 2014, pages 615-625. On drift estimation for non-ergodic fractional Ornstein-Uhlenbeck process with discrete observations.

When $H \in\left(\frac{1}{2}, 1\right)$, it follows from Pipiras and Taqqu (2000) that the elements of $\mathcal{H}$ may not be functions but distributions of negative order. It will be more convenient to work with a subspace of $\mathcal{H}$ which contains only functions. Such a space is the set $|\mathcal{H}|$ of all measurable functions $\varphi$ on $[0, T]$ such that

$$
|\varphi|_{|\mathcal{H}|}^{2}:=H(2 H-1) \int_{0}^{T} \int_{0}^{T}|\varphi(u)||\varphi(v)||u-v|^{2 H-2} d u d v<\infty .
$$

If $\varphi, \psi \in|\mathcal{H}|$ then

$$
E\left[B^{H}(\varphi) B^{H}(\psi)\right]=H(2 H-1) \int_{0}^{T} \int_{0}^{T} \varphi(u) \psi(v)|u-v|^{2 H-2} d u d v .
$$

We know that $\left(|\mathcal{H}|,\langle\cdot, \cdot\rangle_{|\mathcal{H}|}\right)$ is a Banach space, but that $\left(|\mathcal{H}|,\langle\cdot, \cdot\rangle_{\mathcal{H}}\right)$ is not complete (see e.g. Pipiras and Taqqu, 2000). However, we have the dense inclusions $L^{2}([0, T]) \subset L^{\frac{1}{H}}([0, T]) \subset$ $|\mathcal{H}| \subset \mathcal{H}$.For every $q \geq 1$, let $\mathcal{H}_{q}$ be the $q$ th Wiener chaos of $X$, that is, the closed linear subspace of $L^{2}(\Omega)$ generated by the random variables $\left\{H_{q}(X(h)), h \in \mathcal{H},\|h\|_{\mathcal{H}}=1\right\}$, where $H_{q}$ is the $q$ th Hermite polynomial defined as $H_{q}(x)=(-1)^{q} e^{\frac{x^{2}}{2}} \frac{d^{q}}{d x^{q}}\left(e^{-\frac{x^{2}}{2}}\right)$. The mapping $I_{q}\left(h^{\otimes q}\right)=H_{q}(X(h))$ provides a linear isometry between the symmetric tensor product $\mathcal{H}^{\odot q}$ (equipped with the modified norm $\|\cdot\|_{\mathcal{H} \odot q}=\sqrt{q !}\|\cdot\|_{\mathcal{H}^{\otimes q}}$ ) and $\mathcal{H}_{q}$. Specifically, for all $f, g \in \mathcal{H}^{\odot q}$ and $q \geq 1$, one has

$$
E\left[I_{q}(f) I_{q}(g)\right]=q !\langle f, g\rangle_{\mathcal{H} \otimes q} .
$$

The multiple stochastic integral $I_{q}(f)$ satisfies hypercontractivity property:

$$
\left(E\left[\left|I_{q}(f)\right|^{p}\right]\right)^{1 / p} \leqslant c_{p, q}\left(E\left[\left|I_{q}(f)\right|^{2}\right]\right)^{1 / 2} \text { for any } p \geq 2 .
$$

As a consequence, for any $F \in \oplus_{l=1}^{q} \mathcal{H}_{l}$, we have

$$
\left(E\left[|F|^{p}\right]\right)^{1 / p} \leqslant c_{p, q}\left(E\left[|F|^{2}\right]\right)^{1 / 2} \text { for any } p \geq 2 .
$$

\section{Construction and strong consistency of the estimators}

From the explicit solution of (1) which is given by

$$
X_{t}=e^{\theta t} \int_{0}^{t} e^{-\theta s} d B_{s}^{H}
$$

Let us introduce the following processes related to $X_{t}$ :

$$
\xi_{t}:=\int_{0}^{t} e^{-\theta s} d B_{s}^{H}
$$

and

$$
S_{n}:=\Delta_{n} \sum_{i=1}^{n} X_{t_{i-1}}^{2}
$$


So, we can write

$$
\hat{\theta}_{n}=\frac{e^{\theta \Delta_{n}}-1}{\Delta_{n}}+\frac{G_{n}}{S_{n}}
$$

where

$$
G_{n}:=\sum_{i=1}^{n} e^{\theta t_{i}}\left(\xi_{t_{i}}-\xi_{t_{i-1}}\right) X_{t_{i-1}}
$$

We first recall some results of Belfadli et al. (2011) needed throughout the paper:

$$
\lim _{t \rightarrow \infty} \xi_{t}=\xi_{\infty}:=\int_{0}^{\infty} e^{-\theta s} d B_{s}^{H}
$$

almost surely as $t \rightarrow \infty$. Moreover

$$
\sup _{t \geq 0} E\left(\xi_{t}^{2}\right) \leqslant E\left(\xi_{\infty}^{2}\right)=H \Gamma(2 H) \theta^{-2 H}<\infty .
$$

On the other hand

$$
e^{-2 \theta T_{n}} \int_{0}^{T_{n}} X_{t}^{2} d t \longrightarrow \frac{\xi_{\infty}^{2}}{2 \theta}
$$

almost surely as $n \rightarrow \infty$.

For the strong consistency, let us state the following direct consequence of the Borel-Cantelli Lemma (see e.g. Kloeden and Neuenkirch, 2007), which allows us to turn convergence rates in the $p$-th mean into pathwise convergence rates.

Lemma 1. Let $\gamma>0$ and $p_{0} \in \mathbb{N}$. Moreover let $\left(Z_{n}\right)_{n \in \mathbb{N}}$ be a sequence of random variables. If for every $p \geq p_{0}$ there exists a constant $c_{p}>0$ such that for all $n \in \mathbb{N}$,

$$
\left(\mathbb{E}\left|Z_{n}\right|^{p}\right)^{1 / p} \leqslant c_{p} \cdot n^{-\gamma},
$$

then for all $\varepsilon>0$ there exists a random variable $\eta_{\varepsilon}$ such that

$$
\left|Z_{n}\right| \leqslant \eta_{\varepsilon} \cdot n^{-\gamma+\varepsilon} \quad \text { almost surely }
$$

for all $n \in \mathbb{N}$. Moreover, $\mathbb{E}\left|\eta_{\varepsilon}\right|^{p}<\infty$ for all $p \geq 1$.

We will need the following Lemma.

Lemma 2. Let $H \in\left(\frac{1}{2}, 1\right)$. Assume that $\theta>0, \Delta_{n} \rightarrow 0$ and $T_{n} \rightarrow \infty$ as $n \rightarrow \infty$. Then for any $\beta>0$

$$
e^{-2 \theta T_{n}} S_{n}=\frac{\Delta_{n}}{e^{2 \theta \Delta_{n}}-1} \xi_{t_{n-1}}^{2}+o\left(n^{\beta} \Delta_{n}^{H-1} e^{-\theta T_{n}}\right) \quad \text { almost surely. }
$$

In addition, if we assume that $n \Delta_{n}^{1+\alpha} \rightarrow 0$ for some $\alpha>0$,

$$
e^{-2 \theta T_{n}} S_{n}=\frac{\Delta_{n}}{e^{2 \theta \Delta_{n}}-1} \xi_{t_{n-1}}^{2}+o(1) \quad \text { almost surely }
$$

and hence, as $n \rightarrow \infty$

$$
e^{-2 \theta T_{n}} S_{n} \longrightarrow \frac{\xi_{\infty}^{2}}{2 \theta} \quad \text { almost surely }
$$


K. Es-Sebaiy and D. Ndiaye, Afrika Statistika, Vol. 9, 2014, pages 615-625. On drift estimation for non-ergodic fractional Ornstein-Uhlenbeck process with discrete observations.

Proof. Let us start by noting that

$$
\begin{aligned}
e^{-2 \theta T_{n}} S_{n} & =\frac{\Delta_{n}}{e^{2 \theta \Delta_{n}}-1} \sum_{i=1}^{n} e^{-2 \theta(n-i) \Delta_{n}}\left(\frac{e^{2 \theta \Delta_{n}}-1}{e^{2 \theta \Delta_{n}}}\right) \xi_{t_{i-1}}^{2} \\
& =\frac{\Delta_{n}}{e^{2 \theta \Delta_{n}}-1} \sum_{i=1}^{n} e^{-2 \theta(n-i) \Delta_{n}}\left(1-\frac{1}{e^{2 \theta \Delta_{n}}}\right) \xi_{t_{i-1}}^{2} \\
& =\frac{\Delta_{n}}{e^{2 \theta \Delta_{n}}-1} \sum_{i=1}^{n}\left(e^{-2 \theta(n-i) \Delta_{n}}-e^{-2 \theta(n-i+1) \Delta_{n}}\right) \xi_{t_{i-1}}^{2} \\
& =\frac{\Delta_{n}}{e^{2 \theta \Delta_{n}}-1}\left[\xi_{t_{n-1}}^{2}-\sum_{i=2}^{n}\left(\xi_{t_{i-1}}^{2}-\xi_{t_{i-2}}^{2}\right) e^{-2 \theta(n-i+1) \Delta_{n}}\right]
\end{aligned}
$$

Hence

$$
\begin{aligned}
e^{-2 \theta T_{n}} S_{n}-\frac{\Delta_{n}}{e^{2 \theta \Delta_{n}}-1} \xi_{t_{n-1}}^{2} & =\frac{\Delta_{n}}{e^{2 \theta \Delta_{n}}-1}\left[-\sum_{i=2}^{n}\left(\xi_{t_{i-1}}^{2}-\xi_{t_{i-2}}^{2}\right) e^{-2 \theta(n-i+1) \Delta_{n}}\right] \\
: & =\frac{-\Delta_{n}}{e^{2 \theta \Delta_{n}}-1} R_{n}
\end{aligned}
$$

Since

$$
\begin{aligned}
\frac{-\Delta_{n}}{e^{2 \theta \Delta_{n}}-1} & =\frac{-\Delta_{n}}{2 \theta \Delta_{n}+o\left(\Delta_{n}^{2}\right)} \\
& =\frac{-1}{2 \theta+o\left(\Delta_{n}\right)} \\
& =\frac{-1}{2 \theta}+o\left(\Delta_{n}\right),
\end{aligned}
$$

we have

$$
e^{-2 \theta T_{n}} S_{n}-\frac{\Delta_{n}}{e^{2 \theta \Delta_{n}}-1} \xi_{t_{n-1}}^{2}=\left(\frac{-1}{2 \theta}+o\left(\Delta_{n}\right)\right) R_{n} .
$$

From the equality

$$
\sqrt{\Delta_{n}} e^{\theta T_{n}} R_{n}=\sqrt{\Delta_{n}} \sum_{i=1}^{n-1} e^{\theta i \Delta_{n}} e^{-\theta \Delta_{n}(n-i)}\left(\xi_{t_{i}}^{2}-\xi_{t_{i-1}}^{2}\right)
$$

we can write by using Minkowski and Cauchy Schwartz inequalities and (12)

$$
\begin{aligned}
\left(E\left|\sqrt{\Delta_{n}} e^{\theta T_{n}} R_{n}\right|^{2}\right)^{1 / 2} & \leqslant \sqrt{\Delta_{n}} \sum_{i=1}^{n-1} e^{\theta i \Delta_{n}} e^{-\theta \Delta_{n}(n-i)}\left[E\left(\xi_{t_{i}}^{2}-\xi_{t_{i-1}}^{2}\right)^{2}\right]^{1 / 2} \\
& \leqslant 2 \sqrt{\Delta_{n}}\left[E\left(\xi_{\infty}\right)^{2}\right]^{1 / 2} \sum_{i=1}^{n-1} e^{\theta i \Delta_{n}} e^{-\theta \Delta_{n}(n-i)}\left[E\left(\xi_{t_{i}}-\xi_{t_{i-1}}\right)^{4}\right]^{1 / 4} \\
& =2 \sqrt{\Delta_{n}}\left[E\left(\xi_{\infty}\right)^{2}\right]^{1 / 2} \sum_{i=1}^{n-1} e^{\theta i \Delta_{n}} e^{-\theta \Delta_{n}(n-i)}\left[E\left(\xi_{t_{i}}-\xi_{t_{i-1}}\right)^{2}\right]^{1 / 2}
\end{aligned}
$$

Journal home page: www.jafristat.net 
We now calculate

$$
E\left[\left(e^{\theta i \Delta_{n}}\left(\xi_{t_{i}}-\xi_{t_{i-1}}\right)\right)^{2}\right]=H(2 H-1) e^{2 \theta i \Delta_{n}} \int_{(i-1) \Delta_{n}}^{i \Delta_{n}} \int_{(i-1) \Delta_{n}}^{i \Delta_{n}} e^{-\theta s} e^{-\theta r}|s-r|^{2 H-2} d s d r .
$$

Making the change of variables $u=\frac{s}{\Delta_{n}}-i+1$ and $v=\frac{r}{\Delta_{n}}-i+1$ yield

$$
\begin{aligned}
E\left[\left(e^{\theta i \Delta_{n}}\left(\xi_{t_{i}}-\xi_{t_{i-1}}\right)\right)^{2}\right] & =H(2 H-1) \Delta_{n}^{2 H} e^{2 \theta \Delta_{n}} \int_{0}^{1} \int_{0}^{1} e^{-\theta u \Delta_{n}} e^{-\theta v \Delta_{n}}|u-v|^{2 H-2} d u d v \\
& \leqslant H(2 H-1) \Delta_{n}^{2 H} e^{2 \theta \Delta_{n}} \int_{0}^{1} \int_{0}^{1}|u-v|^{2 H-2} d u d v \\
& =\Delta_{n}^{2 H} e^{2 \theta \Delta_{n}} .
\end{aligned}
$$

Therefore

$$
\begin{aligned}
\left(E\left|\sqrt{\Delta_{n}} e^{\theta T_{n}} R_{n}\right|^{2}\right)^{1 / 2} & \leqslant 2 \sqrt{\Delta_{n}} \Delta_{n}^{H} e^{\theta \Delta_{n}}\left[E\left(\xi_{\infty}\right)^{2}\right]^{1 / 2} \sum_{i=1}^{n-1} e^{-\theta \Delta_{n}(n-i)} \\
& =2 \sqrt{\Delta_{n}} \Delta_{n}^{H}\left[E\left(\xi_{\infty}\right)^{2}\right]^{1 / 2}\left(\sum_{i=0}^{n-2} e^{-\theta i \Delta_{n}}\right) \\
& =2 \sqrt{\Delta_{n}} \Delta_{n}^{H}\left[E\left(\xi_{\infty}\right)^{2}\right]^{1 / 2}\left(\frac{1-e^{-\theta(n-1) \Delta_{n}}}{1-e^{-\theta \Delta_{n}}}\right) \\
& \leqslant 2 \sqrt{\Delta_{n}} \Delta_{n}^{H}\left[E\left(\xi_{\infty}\right)^{2}\right]^{1 / 2}\left(\frac{1}{1-e^{-\theta \Delta_{n}}}\right) \\
& =2 \Delta_{n}^{H-1 / 2}\left[E\left(\xi_{\infty}\right)^{2}\right]^{1 / 2}\left(\frac{\Delta_{n}}{1-e^{-\theta \Delta_{n}}}\right) \\
& \leqslant c(H, \theta) \Delta_{n}^{H-1 / 2}
\end{aligned}
$$

where, here and everywhere else, $c(H, \theta)$ is a generic positive constant depending only on $H$ and $\theta$.

Hence for any $\beta>0$

$$
\left(E\left|n^{-\beta} \Delta_{n}^{1-H} e^{\theta T_{n}} R_{n}\right|^{2}\right)^{1 / 2} \leqslant c(H, \theta) n^{-\beta} .
$$

Now, applying (8) and Lemma 1 there exists a random variable $\eta_{\beta}$ such that

$$
\left|\Delta_{n}^{1-H} e^{\theta T_{n}} R_{n}\right| \leqslant\left|\eta_{\beta}\right| n^{\beta / 2} \quad \text { almost surely. }
$$

for all $n \in \mathbb{N}$ with $\mathbb{E}\left|\eta_{\beta}\right|^{p}<\infty$ for all $p \geq 1$.

Thus, the estimation (14) is obtained. For the convergence (15), we suppose that $n \Delta_{n}^{1+\alpha} \rightarrow 0$ for some $\alpha>0$.

Choosing a constant $\gamma>0$ such that $\frac{\beta+1-H}{\gamma}<\alpha$,

$$
n \Delta_{n}^{1+\frac{\beta+1-H}{\gamma}} \rightarrow 0
$$

and by using (14) and the fact that $T_{n}^{\beta+\gamma} e^{-\theta T_{n}} \rightarrow 0$ the estimations (15) and (16) are satisfied. 
Thus we arrive at our main theorem of this section.

Theorem 1. Let $H \in\left(\frac{1}{2}, 1\right)$. Suppose that $\Delta_{n} \rightarrow 0$ and $n \Delta_{n}^{1+\alpha} \rightarrow 0$ as $n \rightarrow \infty$ for some $\alpha>0$. Then, as $n \rightarrow \infty$,

$$
\hat{\theta}_{n} \longrightarrow \theta \quad \text { almost surely, }
$$

and also,

$$
\check{\theta}_{n} \longrightarrow \theta \quad \text { almost surely. }
$$

Proof. We first prove (22). From (10) and (16) it suffices to show that $e^{-2 \theta T_{n}} G_{n}$ converges to 0 almost surely as $n \rightarrow \infty$.

By using (17) we have

$$
\begin{aligned}
\left(E\left|e^{-2 \theta T_{n}} G_{n}\right|^{2}\right)^{1 / 2} & \leqslant e^{-2 \theta T_{n}} \sum_{i=1}^{n} e^{\theta i \Delta_{n}}\left(E X_{t_{i-1}}^{2}\right)^{1 / 2}\left[E\left(\xi_{t_{i}}-\xi_{t_{i-1}}\right)^{2}\right]^{1 / 2} \\
& \leqslant e^{-2 \theta T_{n}} \Delta_{n}^{H} e^{\theta \Delta_{n}} \sum_{i=1}^{n}\left(E X_{t_{i-1}}^{2}\right)^{1 / 2} \\
& \leqslant e^{-2 \theta T_{n}} \Delta_{n}^{H} e^{\theta \Delta_{n}}\left(E \xi_{\infty}^{2}\right)^{1 / 2} \sum_{i=1}^{n} e^{\theta i \Delta_{n}} \\
& \leqslant c(H, \theta) e^{-\theta T_{n}} \Delta_{n}^{H} \frac{1-e^{-\theta T_{n}}}{e^{\theta \Delta_{n}}-1} \\
& \leqslant c(H, \theta) e^{-\theta T_{n}} \Delta_{n}^{H-1}
\end{aligned}
$$

Fix $\beta>0$. Then there exists $\gamma$ a positive constant which verifies (21).

Hence (24) leads to

$$
\left(E\left|e^{-2 \theta T_{n}} G_{n}\right|^{2}\right)^{1 / 2} \leqslant c(H, \theta, \alpha, \beta) n^{-\beta}
$$

By applying (8) and Lemma 1 we conclude that for every $\beta>0$ there exists a random variable $\eta_{\beta}$ such that

$$
\left|e^{-2 \theta T_{n}} G_{n}\right| \leqslant\left|\eta_{\beta}\right| n^{-\beta} \quad \text { almost surely. }
$$

for all $n \in \mathbb{N}$ with $\mathbb{E}\left|\eta_{\beta}\right|^{p}<\infty$ for all $p \geq 1$. Hence, the convergence (22) is proved.

From (4) we can write

$$
\check{\theta}_{n}=\frac{\xi_{T_{n}}^{2}}{2 e^{-2 \theta T_{n}} S_{n}} .
$$

Thus the convergence (23) is a direct consequence of (13) and (16).

\section{Rate consistency of the estimators}

In this section, we will establish that $\sqrt{n \Delta_{n}}\left(\hat{\theta}_{n}-\theta\right)$ and $\sqrt{n \Delta_{n}}\left(\check{\theta}_{n}-\theta\right)$ are tight. 
K. Es-Sebaiy and D. Ndiaye, Afrika Statistika, Vol. 9, 2014, pages 615-625. On drift estimation for non-ergodic fractional Ornstein-Uhlenbeck process with discrete observations.

Theorem 2. Let $H \in\left(\frac{1}{2}, 1\right)$. Assume that $\theta>0, \Delta_{n} \rightarrow 0$ and $n \Delta_{n}^{1+\alpha} \rightarrow \infty$ as $n \rightarrow \infty$ for some $\alpha>0$. Then, for any $q \geq 0$,

$$
\Delta_{n}^{q} e^{\theta T_{n}}\left(\hat{\theta}_{n}-\theta\right) \text { is not tight (equivalently: not bounded in probability). }
$$

In addition, we assume that $n \Delta_{n}^{3} \rightarrow 0$ as $n \rightarrow \infty$. Then the estimator $\hat{\theta}_{n}$ is $\sqrt{T_{n}}-$ consistent, in the sense that the sequence

$$
\sqrt{T_{n}}\left(\hat{\theta}_{n}-\theta\right) \text { is tight. }
$$

Proof. We shall only prove the case where $q=1$. Similarly, we can prove the case where $q>1$, and the case where $0 \leqslant q<1$ is a direct consequence.

From (10) we obtain

$$
\Delta_{n} e^{\theta T_{n}}\left(\widehat{\theta}_{n}-\theta\right)=e^{\theta T_{n}}\left(e^{\theta \Delta_{n}}-1-\theta \Delta_{n}\right)+\frac{\Delta_{n} e^{-\theta T_{n}} G_{n}}{e^{-2 \theta T_{n}} S_{n}} .
$$

Since $n \Delta_{n}^{1+\alpha} \rightarrow \infty$ and $\frac{e^{\theta \Delta_{n}}-1-\theta \Delta_{n}}{\Delta_{n}^{2}} \longrightarrow \theta^{2} / 2$, we deduce that

$$
e^{\theta T_{n}}\left(e^{\theta \Delta_{n}}-1-\theta \Delta_{n}\right) \rightarrow \infty .
$$

By using (24) we have

$$
E\left|\Delta_{n} e^{-\theta T_{n}} G_{n}\right| \leqslant c(H, \theta) \Delta_{n}^{H} \rightarrow 0 .
$$

Combining (28), (29), (30) and (16) we get (26).

Let us now prove (27). We have from (10) that

$$
\sqrt{T_{n}}\left(\hat{\theta}_{n}-\theta\right)=\sqrt{\frac{n}{\Delta_{n}}}\left(e^{\theta \Delta_{n}}-1-\theta \Delta_{n}\right)+\frac{\sqrt{T_{n}} e^{-2 \theta T_{n}} G_{n}}{e^{-2 \theta T_{n}} S_{n}} .
$$

Since $n \Delta_{n}^{3} \rightarrow 0$,

$$
\begin{aligned}
\sqrt{\frac{n}{\Delta_{n}}}\left(e^{\theta \Delta_{n}}-1-\theta \Delta_{n}\right) & =\sqrt{n \Delta_{n}^{3}} \frac{\left(e^{\theta \Delta_{n}}-1-\theta \Delta_{n}\right)}{\Delta_{n}^{2}} \\
& \rightarrow 0
\end{aligned}
$$

On the other hand, the inequality (30) leads to

$$
\begin{aligned}
E\left|\sqrt{T_{n}} e^{-2 \theta T_{n}} G_{n}\right| & \leqslant c(H, \theta) \sqrt{T_{n}^{3}} \Delta_{n}^{H-2} e^{-\theta T_{n}} \\
& \rightarrow 0 .
\end{aligned}
$$

The last convergence comes from $n \Delta_{n}^{3} \rightarrow 0$ and $n \Delta_{n}^{1+\alpha} \rightarrow \infty$.

Consequently, by (31), (32), (33) and (16) we deduce (27).

Theorem 3. Let $H \in\left(\frac{1}{2}, 1\right)$. Suppose that $\Delta_{n} \rightarrow 0$ and $n \Delta_{n}^{1+\alpha} \rightarrow \infty$ as $n \rightarrow \infty$ for some $\alpha>0$. Then, for any $q \geq 0$,

$$
\Delta_{n}^{q} e^{\theta T_{n}}\left(\check{\theta}_{n}-\theta\right) \text { is not tight (equivalently: not bounded in probability). }
$$

In addition, we assume that $n \Delta_{n}^{3} \rightarrow 0$ as $n \rightarrow \infty$. Then the estimator $\check{\theta}_{n}$ is $\sqrt{T_{n}}-$ consistent, in the sense that the sequence

$$
\sqrt{T_{n}}\left(\check{\theta}_{n}-\theta\right) \text { is tight. }
$$


K. Es-Sebaiy and D. Ndiaye, Afrika Statistika, Vol. 9, 2014, pages 615-625. On drift estimation for non-ergodic fractional Ornstein-Uhlenbeck process with discrete observations.

Proof. We shall only prove the case where $q=\frac{1}{2}$. Similarly, we can prove the case where $q>\frac{1}{2}$, and the case where $0 \leqslant q<\frac{1}{2}$ is a direct consequence.

Using the definition of $\check{\theta}_{n}$, we have

$$
\begin{aligned}
\sqrt{\Delta_{n}} e^{\theta T_{n}}\left(\check{\theta}_{n}-\theta\right) & =\sqrt{\Delta_{n}} e^{\theta T_{n}}\left(\frac{X_{t_{n}}^{2}}{2 \Delta_{n} \sum_{i=1}^{n} X_{t_{i-1}}^{2}}-\theta\right) \\
& =\sqrt{\Delta_{n}} e^{\theta T_{n}}\left(\frac{e^{2 \theta T_{n}} \xi_{t_{n}}^{2}}{2 \Delta_{n} \sum_{i=1}^{n} X_{t_{i-1}}^{2}}-\theta\right) \\
& =\frac{\sqrt{\Delta_{n}}}{2} S_{n}^{-1} e^{3 \theta T_{n}}\left(\xi_{t_{n}}^{2}-2 \theta S_{n} e^{-2 \theta T_{n}}\right) .
\end{aligned}
$$

We can write

$$
\begin{aligned}
\sqrt{\Delta_{n}} e^{\theta T_{n}}\left(\check{\theta}_{n}-\theta\right)= & \frac{\sqrt{\Delta_{n}} e^{\theta T_{n}}}{2 e^{-2 \theta T_{n}} S_{n}}\left[\left(\xi_{t_{n}}^{2}-\xi_{t_{n-1}}^{2}\right)+\left(1-\frac{2 \theta \Delta_{n}}{e^{2 \theta \Delta_{n}}-1}\right) \xi_{t_{n-1}}^{2}\right. \\
& \left.-2 \theta\left(e^{-2 \theta T_{n}} S_{n}-\frac{\Delta_{n}}{e^{2 \theta \Delta_{n}}-1} \xi_{t_{n-1}}^{2}\right)\right] .
\end{aligned}
$$

By (17), (18) and (19) we obtain

$$
\begin{aligned}
E\left|\sqrt{\Delta_{n}} e^{\theta T_{n}}\left[\left(\xi_{t_{n}}^{2}-\xi_{t_{n-1}}^{2}\right)-2 \theta\left(e^{-2 \theta T_{n}} S_{n}-\frac{\Delta_{n}}{e^{2 \theta \Delta_{n}}-1} \xi_{t_{n-1}}^{2}\right)\right]\right| & \leqslant c(H, \theta) \Delta_{n}^{H-\frac{1}{2}} \\
& \rightarrow 0 .
\end{aligned}
$$

On the other hand

$$
\begin{aligned}
\sqrt{\Delta_{n}} e^{\theta T_{n}}\left(1-\frac{2 \theta \Delta_{n}}{e^{2 \theta \Delta_{n}}-1}\right) & =\Delta_{n}^{3 / 2} e^{\theta T_{n}}\left(\frac{e^{2 \theta \Delta_{n}-1-2 \theta \Delta_{n}}}{\Delta_{n}^{2}} \frac{\Delta_{n}}{e^{2 \theta \Delta_{n}}-1}\right) \\
& \rightarrow \infty
\end{aligned}
$$

The last convergence comes from the fact that $n \Delta_{n}^{1+\alpha} \rightarrow \infty$ as $n \rightarrow \infty$. Combining (36), (37) and (38) we obtain (34).

Furthermore, using $n \Delta_{n}^{3} \rightarrow 0$ as $n \rightarrow \infty$ the result (35) is obtained.

Remark 1. Assume that $\theta>0$. Belfadli et al. (2011) proved that, in the continuous case, $e^{\theta t}\left(\tilde{\theta}_{t}-\theta\right)$ is asymptotically Cauchy. Then one may also expect that, in the discrete case, $\hat{\theta}_{n}$ and $\check{\theta}_{n}$ are $e^{\theta T_{n}}$-consistent. But the answer is negative, they are $\sqrt{T_{n}}-$ consistent (see Theorem 2 and Theorem 3). 
K. Es-Sebaiy and D. Ndiaye, Afrika Statistika, Vol. 9, 2014, pages 615-625. On drift estimation for non-ergodic fractional Ornstein-Uhlenbeck process with discrete observations.

\section{References}

Belfadli, R. Es-Sebaiy K. and Ouknine, Y. 2011. Parameter Estimation for Fractional Ornstein- Uhlenbeck Processes: Non-Ergodic Case. Frontiers in Science and Engineering (An International Journal Edited by Hassan II Academy of Science and Technology). 1, no. 1, 1-16.

Cénac, P. and Es-Sebaiy K. 2012. Almost sure central limit theorems for random ratios and applications to LSE for fractional Ornstein-Uhlenbeck processes. Arxiv.org/abs/1209.0137.

Dietz, H.M. and Kutoyants,Y.A. 2003. Parameter estimation for some non-recurrent solutions of SDE. Statistics and Decisions 21(1), 29-46.

Jacod, J. 2006. Parametric inference for discretely observed non-ergodic diffusions. Bernoulli 12(3), 383-401.

Hu, Y. and Nualart, D. 2010. Parameter estimation for fractional Ornstein-Uhlenbeck processes. Statistics and Probability Letters 80, 1030-1038.

Kloeden, P. and Neuenkirch, A. 2007. The pathwise convergence of approximation schemes for stochastic differential equations. LMS J. Comp. Math. 10, 235-253.

Nualart, D. 2006. The Malliavin calculus and related topics. Springer-Verlag, Berlin, second edition.

Pipiras, V. and Taqqu, M.S. 2000. Integration questions related to fractional Brownian motion. Probab. Theory Rel. Fields 118, no. 2, 251-291.

Shimizu, Y. 2009. Notes on drift estimation for certain non-recurrent diffusion from sampled data. Statistics and Probability Letters 79, 2200-2207. 